\title{
Atypical Retinal Hemorrhages after Vitrectomy for Idiopathic Macular Hole: Possible Toxicity of Brilliant Blue G
}

\author{
Jae Jung Lee $e^{1,2}$, Seung Kwon Choi ${ }^{1,2}$, Han Jo Kwon ${ }^{1,3}$, Seung Min Lee ${ }^{1,3}$, Sung Who Park ${ }^{1,2}$, Ik Soo Byon ${ }^{1,2}$, Ji Eun Lee ${ }^{1,4}$ \\ ${ }^{1}$ Department of Ophthalmology, Pusan National University School of Medicine, Yangsan, Korea \\ ${ }^{2}$ Biomedical Research Institute, Pusan National University Hospital, Busan, Korea \\ ${ }^{3}$ Research Institute for Convergence of Biomedical Science and Technology, Pusan National University Yangsan Hospital, Yangsan, \\ Korea \\ ${ }^{4}$ Lee Eye Clinic, Busan, Korea
}

Purpose: To describe the development of multiple retinal hemorrhages after uncomplicated macular hole (MH) surgery, and to determine the associated factors.

Methods: The medical records of 163 patients (167 eyes) diagnosed with idiopathic MHs and who underwent surgery at Pusan National University Hospital between March 2016 and July 2018 were retrospectively reviewed. The development of retinal hemorrhages was evaluated using ultra-widefield fundus photographs. Multiple retinal hemorrhages were defined as three or more dot or blot hemorrhages that had not been observed before and during the surgery. The patients were divided into two groups according to the presence of multiple retinal hemorrhages. The variable parameters were compared between the two groups to find the risk factors. The associated factors were evaluated further for the independent factor using multiple logistic regression analysis.

Results: Multiple retinal hemorrhages were observed in 31 eyes (18.6\%) after MH surgery. The associated factors were the surgical induction of posterior vitreous detachment (PVD) $(p=0.003)$, use of the internal limiting membrane flap technique ( $p$ $=0.028)$, and staining with Brilliant Blue G (BBG) $(p=0.003)$. Retinal hemorrhages were exclusively observed in eyes in which BBG was used. Surgical PVD induction was the only independent risk factor (odds ratio, 13.099; $p=0.013$ ). No statistically significant differences were observed between the two groups in the postoperative visual outcomes and MH closure rate. Additionally, patients who underwent surgery for idiopathic epiretinal membrane during the study period were reviewed to validate the above findings. Multiple retinal hemorrhages were noted in only one case $(0.4 \%)$ in which BBG was used after surgical induction of PVD.

Conclusions: Multiple retinal hemorrhages after MH surgery appear to be related to the intravitreal use of BBG in eyes that sustained mechanical damage because of surgical induction of PVD; however, they did not affect surgical outcomes.

Key Words: Brilliant blue G, Retinal hemorrhage, Retinal perforation, Vitrectomy, Vitreous detachment

Received: October 1,2020 Final revision: April 20, 2021

Accepted: April 21, 2021

Corresponding Author: Ji Eun Lee, MD, PhD. Department of Ophthalmology, Pusan National University School of Medicine, 49 Busandaehak-ro, Mulgeum-eup, Yangsan 50612, Korea. Tel: 82-55-360-2131, Fax: 82-55-360-2161, E-mail: jlee@pusan.ac.kr
Idiopathic macular hole $(\mathrm{MH})$ is a vitreomacular interface disorder that causes a full-thickness defect of the neurosensory retina at the fovea and may result in permanent visual impairment [1]. Various techniques that manipulate the internal limiting membrane (ILM), such as ILM peeling [2] or 
ILM flap technique [3], improve the hole-closure rate.

Although pars plana vitrectomy for $\mathrm{MH}$ is a relatively safe and widely performed procedure, various complications have been reported, including peripheral retinal breaks, rhegmatogenous retinal detachment, enlargement of the hole, late reopening of the hole, atrophy of retinal pigment epithelium under the hole, phototoxicity, and endophthalmitis [4]. Manipulation of the ILM is also associated with the risk of undesirable effects. Removal of the ILM may result in postoperative metamorphopsia related to the postoperative deformation of the macula $[5,6]$ and the appearance of a dissociated optic nerve fiber layer [7]. Use of indocyanine green as the vital stain for the ILM was related to functional outcomes inferior to those obtained using Brilliant Blue G (BBG) [8].

The ILM flap technique is gaining popularity for improving the $\mathrm{MH}$ closure rate, and staining of the ILM is essential for the procedure. BBG is one of the safest vital stains currently available, and to our knowledge, no toxicity related to its clinical use has been reported after uncomplicated operations, except the result of accidental contamination in the compounded product [9]. BBG has been marketed in the European Union under the brand name ILM Blue (DORC International BV, Zuidland, The Netherlands) in ready-to-use, prefilled syringes. BBG also received US Food and Drug Administration approval under the brand name TissueBlue (DORC International BV) in 2019 (NDA 209569).

In our clinical practice, we observed multiple retinal hemorrhages that developed in a substantial number of patients after otherwise uncomplicated $\mathrm{MH}$ surgery, possibly related to the use of BBG. Since this phenomenon has not yet been reported to our knowledge, we aimed to analyze its incidence, associated factors, and surgical outcomes in this study.

\section{Materials and Methods}

In this retrospective observational cohort study, we reviewed the medical records of patients diagnosed with idiopathic $\mathrm{MH}$ who underwent surgery at Pusan National University Hospital from March 2016 to July 2018. The study design was approved by the institutional review board of Pusan National University Hospital (1812-006-073), and the study was conducted in accordance with the principles out- lined in the tenets of the Declaration of Helsinki. Informed consent was waived, as the study was conducted retrospectively using the medical records formed from the usual medical practices and treated anonymously.

The existence of retinal hemorrhages was evaluated on wide-angle fundus photographs acquired using an ultra-widefield fundus camera (Optos PLC., Dunfermline, United Kingdom) before surgery and on the day after surgery. Multiple retinal hemorrhages were defined as three or more dot or blot retinal hemorrhages that had not been observed before and during the surgery but only on the day after the surgery. The patients were divided into two groups according to the presence of multiple retinal hemorrhages.

All patients underwent a complete ophthalmic examination, including best-corrected visual-acuity measurement, slit-lamp examination, fundus examination using a 90-diopter lens, widefield fundus photography using the ultra-widefield fundus camera, and spectral-domain optical coherence tomography (OCT; Cirrus HD-OCT, Carl Zeiss Meditec, Dublin, CA, USA) or swept-source OCT (DRIOCT1 Atlantis; Topcon, Tokyo, Japan), pre- and postoperatively. Visual acuity was measured using a Snellen chart and converted to the logarithm of the minimum angle of resolution ( $\log$ MAR) scale for statistical analysis. Age, sex, medical history, surgical induction of posterior vitreous detachment (PVD), adjuvant used for ILM manipulation, vitreous tamponade, and surgical outcomes were reviewed. Postoperative inflammation was assessed according to the Standardization of Uveitis Nomenclature system [10]. Patients' blood pressure before and after the surgery, and the results of preoperative laboratory tests were also reviewed for evaluation of systemic factors.

Two expert surgeons (JEL and SWP) performed the surgeries using similar techniques. A 25-gauge, 3-port pars plana vitrectomy was performed using the Constellation (Alcon Laboratories, Fort Worth, TX, USA) or EVA (DORC International BV) systems. Phacoemulsification was concurrently performed at the surgeon's discretion. Triamcinolone acetonide (MaQaid; Wakamoto Pharmaceutical, Tokyo, Japan) was applied to visualize the vitreous if necessary. If PVD had not occurred after the removal of the core vitreous, the posterior vitreous was separated from the optic disc by aspirating the vitreous with the vitreous cutter. Once PVD developed, it usually expanded to the periphery without additional vitreous cutter aspiration 
during removal of the peripheral vitreous. If the expansion was not sufficient because of strong attachment of the posterior vitreous to the retina, PVD was forcefully expanded beyond the equator by repeated aspiration with the vitreous cutter. The ILM was visualized with BBG (Brilliant Blue G 250; Sigma-Aldrich, St. Louis, MO, USA) or triamcinolone acetonide. BBG was prepared according to a previously reported method, although a different solvent was used due to its availability [11]. BBG was dissolved in a balanced salt solution (Alcon Laboratories). Thereafter, the solution was sterilized using a $0.22-\mu \mathrm{m}$ syringe filter. The final concentration of BBG was $0.25 \mathrm{mg} / \mathrm{mL}$ (290 mOsm, $\mathrm{pH}=7.23$ ). Triamcinolone acetonide was prepared according to the manufacturer's instruction. For visualization of the ILM, 0.2 to $0.3 \mathrm{~mL}$ of the prepared adjuvant was injected into the fluid-filled vitreous cavity and washed out immediately.

ILM peeling and/or the ILM flap technique was used at the surgeon's discretion. The ILM was stained using BBG in all the cases wherein the ILM flap technique was used. The balanced salt solution was replaced by air after sufficient removal of the peripheral vitreous. The air was substituted with non-expansile, long-acting $18 \%$ sulfur hexafluoride gas in certain cases. The sclerotomy sites remained without a suture in most cases but were sutured with 8-0 polyglactin if leakage persisted. All patients were instructed to maintain a face-down position for 1 to 3 days after the surgery.

All statistical analyses were performed using IBM SPSS Statistics ver. 23.0 (IBM Corp., Armonk, NY, USA). The Mann-Whitney $U$-test was used for comparing continuous variables, and the chi-squared test or Fisher exact test was used for comparing categorical variables between the two groups. Preoperative and postoperative values were compared using the paired $t$-test or Wilcoxon signed-rank test. Potential associated factors were evaluated for the independent factor using multiple logistic regression analysis. A $p$-value $<0.05$ was considered statistically significant.

\section{Results}

\section{Fundus findings of multiple retinal hemorrhages}

This study included 167 eyes of 163 patients. Multiple retinal hemorrhages were observed in 31 eyes $(18.6 \%)$ of 30
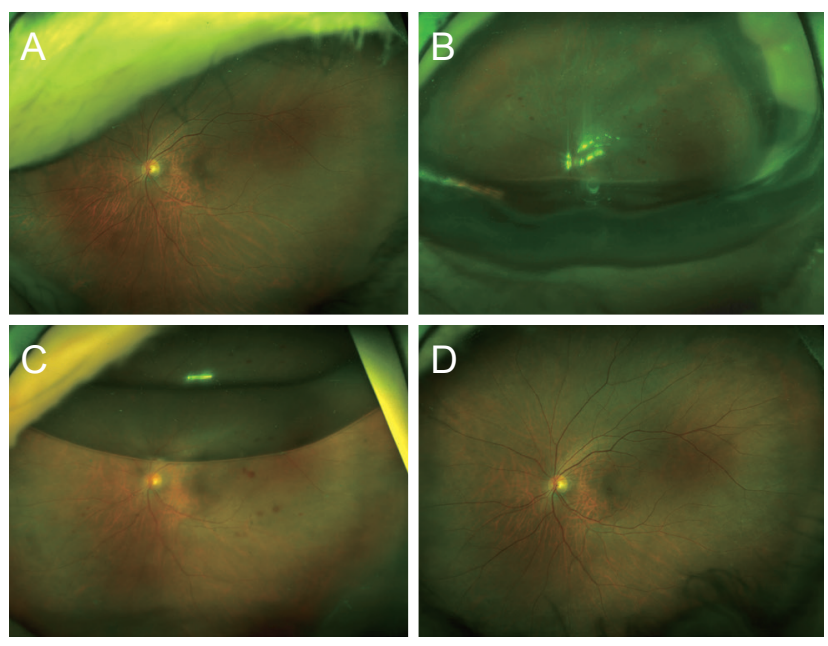

Fig. 1. Representative case from the retinal hemorrhage group. (A) Ultra-widefield fundus photograph of a patient with an idiopathic macular hole before surgery with no other specific finding. (B) A few clusters of retinal hemorrhages in the midperiphery are noted on the widefield fundus photograph acquired the day after uncomplicated macular hole surgery, using Brilliant Blue G stain and a room-air tamponade. (C) After 4 days, the gas bubble remains in less than half of the vitreous cavity, and multiple retinal hemorrhages are clearly observed. (D) The retinal hemorrhages have all resolved at 1 month after the surgery.
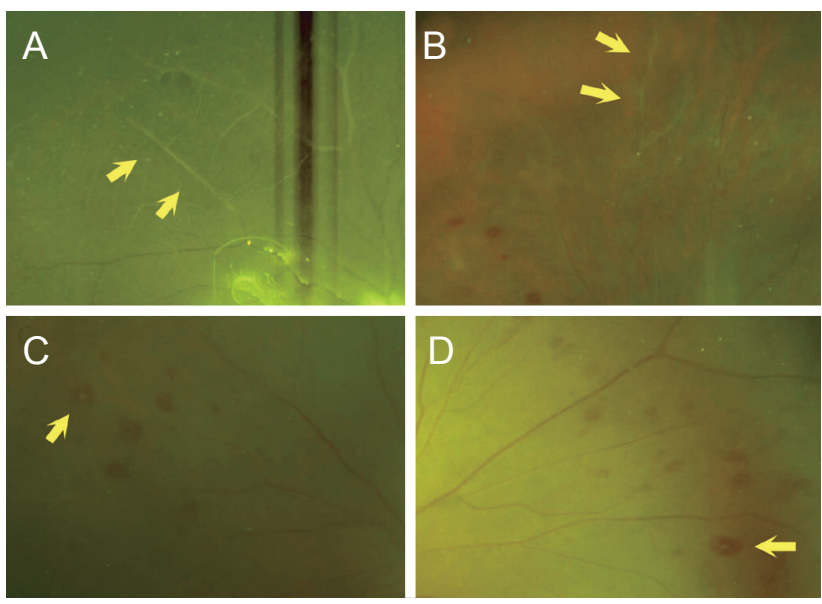

Fig. 2. Various findings of multiple retinal hemorrhages after macular hole surgery. (A,B) Whitening of the retinal vessels (arrows) and (C,D) a central white core, which resembles Roth's spot (arrows), are noted in conjunction with multiple retinal hemorrhages.

patients on the day after otherwise uncomplicated $\mathrm{MH}$ surgery. According to the presence of multiple retinal hemorrhages, the patients were divided into two groups: the hemorrhage and no hemorrhage groups. A representative case is depicted in Fig. 1A-1D, 2A-2D. 
Typically, three or more blot hemorrhages were noted in the midperipheral field except in the macular area. Whitening of the retinal vessels was observed in eight eyes in the hemorrhage group (25.8\%). Some of the hemorrhages had white cores resembling Roth's spots; this was noted in 12 eyes (38.7\%) (Fig. 2).

No patient exhibited worsening of retinal hemorrhages. For all 25 eyes followed up for 3 months or more in the hemorrhage group, retinal hemorrhages spontaneously resolved without further treatment over 1 to 3 months. All eyes also spontaneously recovered from the whitening of the retinal vessels.

\section{Surgical outcomes}

The MH closure rate was $100 \%$ in the hemorrhage group and $98.2 \%$ in the no hemorrhage group ( $p>0.999)$. Anterior chamber cell grading did not significantly differ between the two groups on the day after surgery $(p=0.800)$.

Among 121 eyes for which postoperative visual acuity data were available at 3 months, $25(20.7 \%)$ were in the hemorrhage group and 96 (79.3\%) in the no hemorrhage group. Visual acuity improved significantly in both groups (hemorrhage group, from $0.70 \pm 0.34$ to $0.38 \pm 0.28 \mathrm{log}$ MAR, $p<0.001$; no hemorrhage group, from $0.73 \pm 0.33$ to $0.39 \pm 0.28 \log$ MAR, $p<0.001)$. No statistically significant

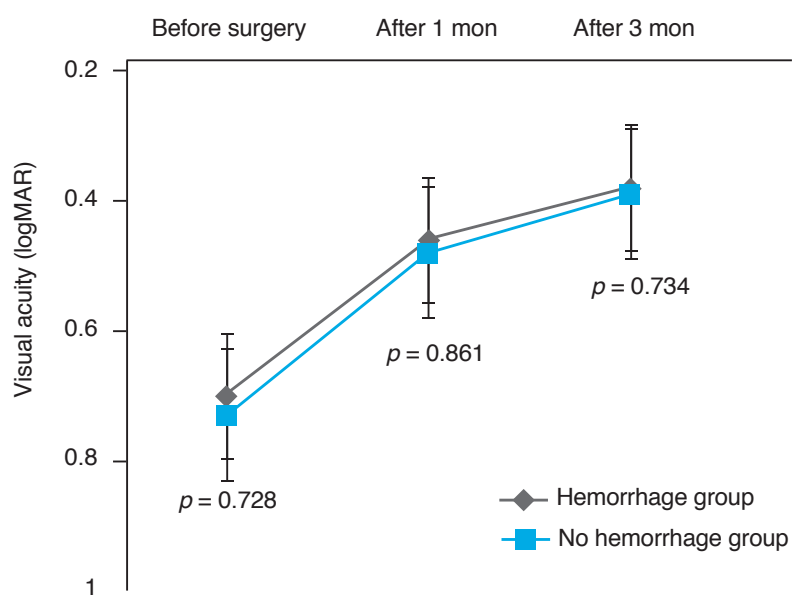

Fig. 3. Changes in visual acuity after macular hole surgery. Visual acuity significantly improved after macular hole surgery ( $p$ $<0.001$ ) in both groups. At 1 and 3 months after the surgery, no statistically significant differences in visual acuity were observed between the hemorrhage and no hemorrhage groups. $\log \mathrm{MAR}=$ logarithm of the minimum angle of resolution. differences were observed in postoperative visual acuity and visual-acuity changes between the two groups (Fig. 3).

\section{Risk factors}

No differences were observed between the two groups in the baseline characteristics, including age, sex, medical history, laterality, preoperative visual acuity, MH size, blood pressure, laboratory tests, or anti-platelet medication (Table 1). Surgical induction of PVD was significantly more frequent in the hemorrhage group (30 eyes, 96.8\%) than in the no hemorrhage group (101 eyes, $74.3 \%, p=$ 0.004). The ILM flap technique was used more frequently in the hemorrhage than in the no hemorrhage group $(77.4 \%$ vs. $56.6 \%, p=0.041)$. BBG was used as the ILM staining material in all the cases in the hemorrhage group (100.0\%), whereas it was used in $79.4 \%$ of cases in the no hemorrhage group ( $p=0.003)$.

Multiple logistic regression analysis revealed that the surgical induction of PVD was the only independent risk factor for the development of multiple retinal hemorrhages ( $p=0.013$; odds ratio, 13.099) (Table 2).

\section{Supplemental studies}

To evaluate the cause of multiple retinal hemorrhages, fluorescein angiography was performed in selected cases in the hemorrhage group at postoperative days 1 to 7 . Although the image quality was poor because of the remaining gas in the vitreous cavity, fluorescein angiography revealed blocked fluorescence at the sites of the hemorrhages. No vascular leakage or non-perfusion was observed at the lesion sites (Fig. 4A-4E).

To investigate the association among the development of multiple retinal hemorrhages, using BBG and surgical induction of PVD, patients who underwent surgery for idiopathic epiretinal membrane (ERM) during the study period were reviewed $(n=280)$. BBG was used to stain the ILM in 188 eyes (67.1\%). Multiple retinal hemorrhages were noted in only one eye $(0.4 \%)$ with ERM. In that case, the surgical technique was consistent with that used in the hemorrhage group in this study; PVD was induced surgically using the vitreous cutter, and BBG was applied to stain the ILM. 
Table 1. Comparison of baseline characteristics, surgical methods, and systemic factors

\begin{tabular}{|c|c|c|c|}
\hline & Hemorrhage group $(\mathrm{n}=31)$ & No hemorrhage group $(n=136)$ & $p$-value \\
\hline Age (yr) & $64.8 \pm 7.1$ & $65.4 \pm 10.4$ & 0.746 \\
\hline Male : female & $11: 20$ & $47: 89$ & $>0.999$ \\
\hline \multicolumn{4}{|l|}{ Underlying disease } \\
\hline Hypertension & $8(25.8)$ & $51(37.5)$ & 0.298 \\
\hline DM & $2(6.5)$ & $21(15.4)$ & 0.255 \\
\hline Cardiac disease & $1(3.2)$ & $11(8.1)$ & 0.698 \\
\hline Renal disease & $3(9.7)$ & $6(4.4)$ & 0.370 \\
\hline Liver disease & $0(0)$ & $6(4.4)$ & 0.594 \\
\hline Anti-platelet medication & $1(3.2)$ & $14(10.3)$ & 0.309 \\
\hline Laterality (right : left) & $14: 17$ & $67: 69$ & 0.696 \\
\hline \multicolumn{4}{|l|}{ Visual acuity } \\
\hline Median (Snellen) & $20 / 100$ & $20 / 100$ & \multirow{2}{*}{0.601} \\
\hline Mean (logMAR) & $0.72 \pm 0.32$ & $0.76 \pm 0.35$ & \\
\hline Hole size $(\mu \mathrm{m})$ & $472.4 \pm 196.4$ & $441.4 \pm 203.2$ & 0.241 \\
\hline Surgical induction of PVD $(+/-)$ & $30 / 1$ & $101 / 35$ & $0.004^{*}$ \\
\hline ILM peeling : ILM flap technique & $7: 24$ & $59: 77$ & $0.041^{*}$ \\
\hline ILM staining (BBG : TA) & $31: 0$ & $108: 28$ & $0.003^{*}$ \\
\hline Vitreous tamponade (air : $18 \% \mathrm{SF}_{6}$ ) & $31: 0$ & $129: 7$ & 0.351 \\
\hline Preoperative SBP (mmHg) & $121.9 \pm 13.8$ & $119.3 \pm 12.6$ & 0.310 \\
\hline Preoperative DBP (mmHg) & $73.6 \pm 8.4$ & $74.5 \pm 8.6$ & 0.569 \\
\hline Postoperative SBP (mmHg) & $119.4 \pm 16.1$ & $122.9 \pm 13.2$ & 0.193 \\
\hline Postoperative DBP (mmHg) & $73.6 \pm 10.2$ & $76.2 \pm 9.2$ & 0.161 \\
\hline \multicolumn{4}{|l|}{ Laboratory findings } \\
\hline $\mathrm{Hb}(\mathrm{g} / \mathrm{dL})$ & $13.6 \pm 1.1$ & $13.5 \pm 1.4$ & 0.800 \\
\hline $\mathrm{WBC}\left(10^{3} / \mu \mathrm{L}\right)$ & $6.5 \pm 2.0$ & $6.2 \pm 1.7$ & 0.500 \\
\hline Platelet $\left(10^{3} / \mu \mathrm{L}\right)$ & $255.6 \pm 68.6$ & $255.7 \pm 63.4$ & 0.989 \\
\hline AST : ALT ratio & $1.1 \pm 0.3$ & $1.2 \pm 0.3$ & 0.192 \\
\hline T. Chol (mg/dL) & $195.9 \pm 43.6$ & $187.8 \pm 40.6$ & 0.326 \\
\hline BUN : Cr ratio & $22.2 \pm 7.1$ & $20.9 \pm 6.9$ & 0.345 \\
\hline PT time (sec) & $11.3 \pm 0.6$ & $11.4 \pm 1.0$ & 0.505 \\
\hline aPTT time (sec) & $31.5 \pm 4.3$ & $32.6 \pm 7.8$ & 0.461 \\
\hline
\end{tabular}

Values are presented as mean \pm standard deviation, number $(\%)$ unless otherwise indicated.

$\mathrm{DM}=$ diabetes mellitus; $\log \mathrm{MAR}=$ logarithm of the minimum angle of resolution; $\mathrm{PVD}=$ posterior vitreous detachment; ILM = internal limiting membrane; $\mathrm{BBG}=$ Brilliant Blue $\mathrm{G} ; \mathrm{TA}=$ triamcinolone acetonide; $\mathrm{SF}_{6}=$ sulfur hexafluoride; $\mathrm{SBP}=$ systolic blood pressure; $\mathrm{DBP}$ = diastolic blood pressure; $\mathrm{Hb}=$ hemoglobin; $\mathrm{WBC}=$ white blood cell; AST = aspartate aminotransferase; ALT = alanine aminotransferase; $\mathrm{T}$. $\mathrm{Chol}=$ total cholesterol; $\mathrm{BUN}=$ blood urea nitrogen $; \mathrm{Cr}=$ creatinine; $\mathrm{PT}=$ prothrombin; $\mathrm{aPTT}=$ activated partial thromboplastin . ${ }^{*} p<0.05$ was considered statistically significant.

\section{Discussion}

In the present study, we explored novel findings of multiple retinal hemorrhages, documented using ultra-widefield fundus photography, in $18.6 \%$ of cases on the day after otherwise uncomplicated MH surgery. This complication was related to the surgical induction of PVD and use of BBG. The hemorrhages resolved within several months sponta- 
Table 2. Multiple regression analysis of factors associated with the development of multiple retinal hemorrhages

\begin{tabular}{lccc}
\hline & OR & $95 \%$ CI & $p$-value \\
\hline Surgical induction of PVD $(+/-)$ & 13.099 & $1.709-100.389$ & $0.013^{*}$ \\
ILM peeling : ILM flap technique & 1.358 & $0.438-4.208$ & 0.596 \\
ILM staining (BBG : TA) & NA & NA & 0.998 \\
\hline
\end{tabular}

$\mathrm{OR}=$ odds ratio; $\mathrm{CI}=$ confidence interval; $\mathrm{PVD}=$ posterior vitreous detachment; $\mathrm{ILM}=$ internal limiting membrane; $\mathrm{BBG}=\mathrm{Brilliant}$ Blue G; TA = triamcinolone acetonide; NA = not applicable. ${ }^{*} p<0.05$ was considered statistically significant.
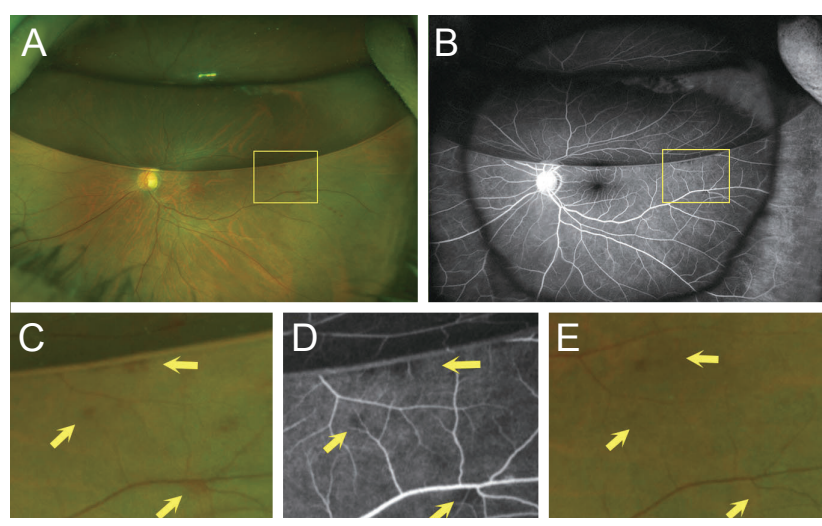

Fig. 4. Fluorescein angiography findings of multiple retinal hemorrhages after macular hole surgery. (A) Multiple retinal hemorrhages are noted in a 65 -year-old male 6 days after macular hole surgery. (B) Fluorescein angiography reveals blocked fluorescence at the sites of hemorrhage, without leakage or non-perfusion. (C) Multiple blot hemorrhages are observed in the area temporal to the macula (arrows). (D) Blocked fluorescence is noted at the sites of hemorrhage (arrows). (E) The hemorrhages are almost completely resolved at six weeks after surgery (arrows).

neously, leaving no apparent sequelae.

The recent interest in these findings would be related to the introduction of BBG and ultra-widefield fundus photography. Poor visibility of the retinal periphery in a gasfilled eye may prevent the detection of small retinal hemorrhages. If long-acting gas is used as tamponade, the retinal hemorrhages may resolve before the complete absorption of the gas. We recognized the phenomena because of our frequent use of room-air tamponade. In particular, ultra-widefield fundus photography provides high-resolution images of the periphery of gas-filled eyes, which assisted us in retrospectively detecting the subtle findings of retinal hemorrhages and vascular whitening.

As multiple retinal hemorrhages after MH surgery were, to our knowledge, not reported previously, various possible mechanisms were speculated based on the available clinical data. When we first noticed such hemorrhages in clini- cal practice, we suspected endophthalmitis $[12,13]$. However, this was ruled out as none of the cases progressed to infectious endophthalmitis. Fluorescein angiography revealed no vascular leakage or non-perfusion, which led us to exclude retinal vascular obstruction and vasculitis. The possibility of toxicity of the gas was also low because room air was used as the vitreous substitute in most cases in this study, and similar findings were not detected after retinal detachment surgery in the same period.

Statistical analysis revealed that surgical induction of PVD, use of BBG, and use of the ILM flap technique were statistically significantly more frequent in the hemorrhage group than in the no hemorrhage group. The higher proportion of the ILM flap technique could be explained with the fact that BBG was used more frequently with that technique than with simple ILM peeling. Accordingly, we suggest that surgical induction of PVD and use of BBG are possible mechanisms accounting for the occurrence of postoperative retinal hemorrhages.

Surgical induction of PVD was performed more frequently in the hemorrhage group than in the no hemorrhage group and was the only independent risk factor for the development of multiple retinal hemorrhages. The pathophysiology of PVD involves the separation of the vitreous cortex from the ILM of the retina [14]. The vitreous is firmly attached physiologically to several structures, including the retinal vessels [15], and hemorrhage is one of the complications after spontaneous development of PVD [16]. The occurrence of $\mathrm{MH}$ is pathophysiologically related to strong vitreoretinal adhesion $[17,18]$. This supports the theory of mechanical damage to the retinal vessels caused by surgical induction of PVD. This hypothesis is also supported by the low incidence of multiple retinal hemorrhages after vitrectomy for ERM, wherein PVD was already present before the surgery in most cases. However, patients in the no hemorrhage group did not exhibit postoperative multiple retinal hemorrhages despite a considerable num- 
ber of them undergoing surgical PVD induction. We speculate that the use of BBG is another key factor for the development of postoperative retinal hemorrhages, as this complication occurred exclusively in the eyes in which BBG was used during the surgery.

An experimental study in which BBG was used in vitro suggested that the exposure of retinal glial cells to clinically relevant concentrations of BBG did not cause cellular apoptosis, and that BBG was less toxic than indocyanine green [19]. BBG had a protective effect on human retinal pigment epithelium in vitro, and on neurons in an experimental animal model $[20,21]$. Clinically, BBG is known as a relatively safe vital dye for intravitreal usage [11,22] and resulted in better functional outcomes than did indocyanine green when used for staining the ILM [8]. Nevertheless, BBG toxicity has been reported in the clinical setting. In two cases of BBG-related complications during vitreoretinal surgery, BBG had accidentally migrated into the subretinal space [23,24]. Drainage was not attempted as the fovea was unaffected, and retinal pigment epithelium atrophy was observed in the area of subretinal migration [23]. A comparative study on the use of BBG in the anterior chamber reported that the corneal endothelial count was significantly lower in the group with BBG staining of the anterior capsule than in the group without BBG staining [25].

In our study, BBG was prepared following a report by Enaida et al. [11], except that balanced salt solution (290 mOsm) was used as solvent instead of OPEGUARD-MA (289 mOsm; Senjyu Pharmaceutical, Osaka, Japan). As the incidence of hemorrhage was very low $(0.4 \%)$ after ERM surgery where the ILM was stained using the BBG solution prepared with the same protocol, it is not plausible that the observed multiple retinal hemorrhages were due to a preparation error.

Based on a comprehensive review of the analyzed results of the current series and the related literature, we suggest the following mechanism. 1) The surgical induction of PVD caused mechanical damage to the superficial retinal surfaces and retinal vessels. 2) The damaged retinal vessels were subsequently exposed to the injected BBG, and BBG toxicity caused retinal hemorrhage as observed in the ultra-widefield fundus photographs. In other words, for the development of BBG toxicity, the preceding damage to the retinal vessels by PVD induction was required. Thus, we hypothesize that surgical induction of PVD was the independent risk factor and use of BBG was a dependent risk factor for multiple retinal hemorrhages following MH surgery. This hypothesis is consistent with the observation that multiple hemorrhages were rare after ERM surgery. The whitening of the retinal vessels also supports our hypothesis of vessel damage due to BBG toxicity.

The multiple retinal hemorrhages did not affect postoperative visual acuity and all the patients exhibited statistically significant visual improvement. This may be explained by the occurrence of the hemorrhages in the midto far periphery and not in the macula. As documented in an OCT study on the progress of idiopathic MH development, the posterior vitreous is separated from the macula in patients with MHs, even in the presence of vitreopapillary adhesion [26], and surgical induction of PVD will not affect the macula related to the vitreoretinal adhesion. In addition, damage to the macular retinal vessels by ILM peeling occurs after staining with BBG and will not result in the development of postoperative retinal hemorrhage, according to our proposed theory.

The limitations of the current study are its retrospective design, single-center nature, and short follow-up period, as well as the relatively small number of cases in which fluorescein angiography was performed. However, we reviewed postoperative ultra-widefield photographs in a relatively large number of cases of idiopathic MH and ERM, and documented novel findings, outcomes, and possible mechanisms.

In summary, multiple retinal hemorrhages may develop the day after uncomplicated MH surgery, and gradually resolve with no additional work-up or treatment, and without affecting anatomical or functional outcomes. This complication was observed only in eyes in which the ILM was stained using BBG after surgical induction of PVD. Atypical retinal hemorrhage after MH surgery seems to be related to the intravitreal use of BBG when the retinal vessels are damaged mechanically by PVD induction.

\section{Conflict of Interest}

Ji Eun Lee has the following disclosures to make: Bayer (consultant, honoraria, travel grant, and research fund), Novartis (consultant, honoraria, and research fund), Allergan (consultant and honoraria), Alcon (honoraria), and Abbvie (consultant and honoraria). The other authors have no potential conflict of interest to this article. 


\section{References}

1. la Cour M, Friis J. Macular holes: classification, epidemiology, natural history and treatment. Acta Ophthalmol Scand 2002;80:579-87.

2. Mester V, Kuhn F. Internal limiting membrane removal in the management of full-thickness macular holes. Am J Ophthalmol 2000;129:769-77.

3. Shin MK, Park KH, Park SW, et al. Perfluoro-n-octane-assisted single-layered inverted internal limiting membrane flap technique for macular hole surgery. Retina 2014;34: 1905-10.

4. Park SS, Marcus DM, Duker JS, et al. Posterior segment complications after vitrectomy for macular hole. Ophthalmology 1995;102:775-81.

5. Kim JH, Kang SW, Park DY, et al. Asymmetric elongation of foveal issue after macular hole surgery and its impact on metamorphopsia. Ophthalmology 2012;119:2133-40.

6. Park SH, Park KH, Kim HY, et al. Square grid deformation analysis of the macula and postoperative metamorphopsia after macular hole surgery. Retina 2021;41:931-39.

7. Ito Y, Terasaki H, Takahashi A, et al. Dissociated optic nerve fiber layer appearance after internal limiting membrane peeling for idiopathic macular holes. Ophthalmology 2005;112:1415-20.

8. Baba T, Hagiwara A, Sato E, et al. Comparison of vitrectomy with brilliant blue $\mathrm{G}$ or indocyanine green on retinal microstructure and function of eyes with macular hole. Ophthalmology 2012;119:2609-15.

9. Mikosz CA, Smith RM, Kim M, et al. Fungal endophthalmitis associated with compounded products. Emerg Infect Dis 2014;20:248-56.

10. Jabs DA, Nussenblatt RB, Rosenbaum JT; Standardization of Uveitis Nomenclature (SUN) Working Group. Standardization of uveitis nomenclature for reporting clinical data. Results of the First International Workshop. Am J Ophthalmol 2005;140:509-16.

11. Enaida H, Hisatomi T, Hata Y, et al. Brilliant blue G selectively stains the internal limiting membrane/brilliant blue G-assisted membrane peeling. Retina 2006;26:631-6.

12. Godley BF, Folk JC. Retinal hemorrhages as an early sign of acute bacterial endophthalmitis. Am J Ophthalmol 1993;116:247-9.

13. Relhan N, Jalali S, Nalamada S, et al. Traumatic endoph- thalmitis presenting as isolated retinal vasculitis and white-centered hemorrhages: case report and review of literature. Indian J Ophthalmol 2012;60:317-9.

14. Hogan MJ. The vitreous, its structure, and relation to the ciliary body and retina. Proctor award lecture. Invest Ophthalmol 1963;2:418-45.

15. Spencer LM, Foos RY. Paravascular vitreoretinal attachments. Role in retinal tears. Arch Ophthalmol 1970;84:55764.

16. Witmer MT, Cohen SM. Oral anticoagulation and the risk of vitreous hemorrhage and retinal tears in eyes with acute posterior vitreous detachment. Retina 2013;33:621-6.

17. Smiddy WE, Flynn HW Jr. Pathogenesis of macular holes and therapeutic implications. Am J Ophthalmol 2004;137:52537.

18. Kim HW, Lee JE. Is macular hole a risk factor for retinal detachment? Eye Sci 2015;30:134-6.

19. Kawahara S, Hata Y, Miura M, et al. Intracellular events in retinal glial cells exposed to ICG and BBG. Invest Ophthalmol Vis Sci 2007;48:4426-32.

20. Awad D, Schrader I, Bartok M, et al. Brilliant Blue G as protective agent against trypan blue toxicity in human retinal pigment epithelial cells in vitro. Graefes Arch Clin Exp Ophthalmol 2013;251:1735-40.

21. Wang XH, Xie X, Luo XG, et al. Inhibiting purinergic $\mathrm{P} 2 \mathrm{X} 7$ receptors with the antagonist brilliant blue $\mathrm{G}$ is neuroprotective in an intranigral lipopolysaccharide animal model of Parkinson's disease. Mol Med Rep 2017;15:768-76.

22. Remy M, Thaler S, Schumann RG, et al. An in vivo evaluation of Brilliant Blue $\mathrm{G}$ in animals and humans. $\mathrm{Br} \mathrm{J} \mathrm{Oph-}$ thalmol 2008;92:1142-7.

23. Malerbi FK, Maia M, Farah ME, Rodrigues EB. Subretinal brilliant blue $\mathrm{G}$ migration during internal limiting membrane peeling. Br J Ophthalmol 2009;93:1687.

24. Almeida FP, De Lucca AC, Scott IU, et al. Accidental subretinal brilliant blue $\mathrm{G}$ migration during internal limiting membrane peeling surgery. JAMA Ophthalmol 2015;133:85-8.

25. Park YM, Park JY, Lee JS, et al. Comparative analysis of brilliant blue $\mathrm{G}$ and an intracameral illuminator in assisting visualization of the anterior capsule in eyes with vitreous hemorrhage. J Cataract Refract Surg 2016;42:1015-21.

26. Chauhan DS, Antcliff RJ, Rai PA, et al. Papillofoveal traction in macular hole formation: the role of optical coherence tomography. Arch Ophthalmol 2000;118:32-8. 